\title{
La atención Odontológica a Pacientes COVID-19 Positivo ¿Qué hacer ante una Urgencia?
}

\author{
Dental Care for COVID-19 Positive Patients. What to do in an Emergency?
}

\author{
Camila Boin Bakit ${ }^{1}$ \& Andrés Melián Rivas ${ }^{2,3}$
}

BOIN, B. C. \& MELIÁN, R. A. La atención odontológica a pacientes COVID-19 positivo ¿Qué hacer ante una urgencia?. Int. J. Odontostomat., 14(3):321-324, 2020.

RESUMEN: El pasado diciembre en China, se originó un brote de Neumonía producida por una nueva cepa viral de coronavirus o SARS-CoV-2, nunca antes vista en humanos, lo que despertó la atención en el mundo. Asimismo, el 30 de enero fue declarada una emergencia de salud pública de preocupación internacional por la OMS, marcando el inicio de una pandemia altamente patógena denominada "enfermedad por coronavirus del 2019 (COVID-19)" que se transmite de persona a persona por medio de secreciones respiratorias. Los principales síntomas que se presentan en los pacientes son: fiebre, tos, dolor de garganta, dificultad respiratoria, fatiga y malestar general; sin embargo existe un porcentaje de personas asintomáticas que representan un alto riesgo de contagio para los profesionales de la salud que trabajan en el área buco-nasal como lo son los cirujanos dentistas. En consecuencia, este estudio tiene como objetivo mostrar las medidas de protección que han sido exitosas en otros países para la atención de personas con COVID-19, de manera que los procedimientos sean realizados de una forma segura tanto para los pacientes como para el equipo de salud, evitando la propagación de la enfermedad.

PALABRAS CLAVE: COVID-19, Odontología, Protocolos, Seguridad.

\section{INTRODUCCIÓN}

En diciembre de 2019 en Wuhan, China, se registró por primera vez la enfermedad por coronavirus (COVID-19), causada por un beta-coronavirus altamente contagioso. En un periodo corto de tiempo COVID-19 se propagó desde Asia, Europa, América y finalmente por todo el mundo. Lo anterior se explica debido a su número reproductivo $(\mathrm{R} 0=2.8)$ y a su rápido tiempo de duplicación (6.4 días), además de presentar un pródromo asintomático altamente infeccioso (Meng et al., 2020; Chan et al., 2020a).

La mayor parte de los pacientes infectados (81 $\%)$ cursaron asintomáticos o con síntomas leves mientras que el $15 \%$ requirió hospitalización por su estado de gravedad en China. Además, se ha descrito que la transmisión se produce por pacientes sintomáticos, pero también se ha reportado que los pacientes asintomáticos o en periodo de incubación (14 días o más) pueden ser una fuente de transmisión (Wu \& McGoogan, 2020).
Estos últimos mencionados, presentan un riesgo real para los profesionales de la salud que manejan áreas digestivas y respiratorias tales como: médicos especialistas en enfermedades respiratorias, otorrinolaringólogos, gastroenterólogos, terapeutas respiratorios, cirujanos de cabeza y cuello, cirujanos maxilofaciales y odontólogos, siendo el personal más susceptible a infectarse de COVID-19 (Meng et al.; Kowalski et al., 2020; Ran et al., 2020; Lai et al., 2020). De hecho, la primera muerte de un personal de la salud por COVID-19 en Wuhan, fue un otorrinolaringólogo el 25 de enero de 2020 (Chan et al., 2020b). Además, los trabajadores de la salud representaron el $3.8 \%$ de los infectados en China, mientras que en Italia fue del $20 \%$ (Remuzzi \& Remuzzi, 2020). Donde es importante considerar, que en China se tomaron medidas estrictas tales como cuarentenas totales, uso universal de mascarillas, testeos masivos y establecimientos diferenciados para pacientes con COVID-19 (Coulthard, 2020).

\footnotetext{
${ }^{1}$ Cirujana Dentista, Facultad de Odontología, Universidad de Chile, Chile.

${ }^{2}$ Cirujano Dentista, Pasante Servicio de Cirugía Maxilofacial, Hospital San Juan de Dios, Santiago, Chile.

${ }^{3}$ Docente instructor, Anatomía Humana Normal, Facultad de Medicina, Escuela de Odontología, Universidad del Desarrollo, Santiago, Chile.
} 
Particularmente en Chile, la curva de contagiados va en ascenso, por lo que existe una alta probabilidad de que pacientes diagnosticados con COVID-19 o pacientes con esta condición pero asintomáticos, puedan requerir atenciones bucodentales de urgencia, ya sea dolorosa y/o infecciosa, aumentando el riesgo de contagio por parte del equipo odontológico.

\section{Protocolos descritos}

En estado de pandemia, es importante implementar protocolos efectivos con la finalidad de proteger a los pacientes y al equipo de salud. De acuerdo a lo reportado, se deben ejecutar protocolos de bioseguridad de forma universal en todo establecimiento de salud, tanto del sector público como privado, dado a que un porcentaje importante de los pacientes infectados por COVID-19 son asintomáticos (Bartoszko et al.,), siendo rigurosos con todas las personas atendidas. En el área odontológica especialmente, es frecuente la producción de aerosoles, quedando gotas en las salas de procedimientos, hasta por 3 horas posterior a la atención (Meng et al.; Li et al., 2020; van Doremalen et al., 2020), siendo los pacientes de atenciones posteriores y el equipo de salud susceptibles a contagio, con un alto riesgo de contaminación.

Algunas medidas de bioseguridad implementadas que resultaron exitosas se reportaron desde la Unidad de emergencia dental en la Escuela y Hospital de Estomatología en Wuhan, donde se trataron a más de 700 pacientes con urgencias dentales sin reportar ninguna infección por COVID-19 en el personal de salud (Meng et al.). Entre ellas se describieron las siguientes:

a) Estaciones de Triage: Se registró la temperatura de todo el personal, de los pacientes y sus acompañantes, junto con la entrega continua de mascarillas a cada persona.

b) Se evitaron técnicas de producción de aerosoles o minimización de ellas.

c) Uso de gomas diques y eyectores de salivas de alto volumen para minimizar los aerosoles y salpicaduras durante los procedimientos quirúrgicos.

d) Trabajo a cuatro manos.

e) Estricto uso de elementos de protección personal: Protectores oculares, mascarillas, protectores faciales, impermeable de aislamiento de contacto y guantes.

Mientras que en el área médica, el personal que trabaja con la vía aérea y digestiva en pacientes con
COVID-19 donde se ve expuesto a secreciones, sangre, gotitas y aerosoles (al igual que los odontólogos) trabajan estrictamente:

a) En una sala de aislamiento (Zou et al., 2020; Zuo et al., 2020) o quirófano con entorno de presión negativa (Ti et al., 2020)

b) Con protección personal especial anti-fluidos, mascarillas $\mathrm{N}^{\circ} 95$, gorro, bata, guantes, protectores faciales, anteojos anti empañamiento, cubrezapatos, y si está disponible, una capucha protectora (Zou et al.; Zuo et al.).

c) Y en cuanto a los instrumentos utilizados:

- Todas las herramientas de manejo de la vía aérea deben ser desechables (Zuo et al.; Zou et al.). - Todos los dispositivos deben recogerse en bolsas con doble sellado y la desinfección adecuada implementada durante la eliminación (Zuo et al.; Zou et al.)

- Todos los equipos y las superficies del entorno deben limpiarse y desinfectarse (Zuo et al.; Zou et al.).

\section{Uso de mascarillas}

Es importante entender el uso correcto de elementos de protección respiratoria, ya que estos son críticos para la seguridad del equipo de salud con alto riesgo de contraer COVID-19, tales como los odontólogos. Sin embargo, las recomendaciones existentes tienden a ser contradictorias sobre la eficiencia de las medidas de protección contra este virus. La literatura ha reportado que las mascarillas médicas (entiéndase como mascarillas quirúrgicas, de procedimiento, de aislamiento, láser, resistentes a fluidos o faciales certificadas para uso como dispositivo médico) en comparación con las mascarillas N95 [definidas como mascarilla filtrante N95 certificados por el Instituto Nacional de Seguridad Ocupacional y Salud (NIOSH) y mascarilla con filtro estándar europeo (FFP2)] (Smith et al., 2016), ofrecen una protección similar contra la infección viral respiratoria durante la atención que no genera aerosoles (Bartoszko et al.,). Sin embargo muchas de estas aseveraciones hacen alusión a procedimientos médicos y no al área odontológica (Coulthard).

Teóricamente, los aerosoles son partículas sólidas y líquidas suspendidas en el aire durante tiempos prolongados de un tamaño menor a $50 \mathrm{~mm}$ de diámetro. Por otro lado, la salpicadura es una mezcla de aire, agua y/o sustancias sólidas (de aproximadamente $50 \mathrm{~mm}$ a varios milímetros de diámetro). Las 
mascarillas quirúrgicas presentan una tasa de filtración de alrededor del $80 \%$, mientras que las N95 ofrece una tasa de filtración del $99 \%$ de todas las partículas que miden hasta $0,6 \mathrm{~mm}$. Si comparamos información con el diámetro de COVID-19, que es de $120 \mathrm{~nm}(0,12$ $\mathrm{mm}$ ) (Health and Safety Executive, 2020; Coulthard) la mascarilla N95 protegería en mayor medida en caso de realizar procedimientos con aerosoles.

Para los procedimientos generadores de aerosoles, se recomiendan las mascarillas N95 por unanimidad según las pautas internacionales. En contraste, las atenciones de rutina o procedimientos que no generan aerosoles presentan inconsistencias en los pacientes con COVID-19. Mientras la OMS recomienda el uso de mascarillas médicas, por su seguridad, costo y disponibilidad, el Centro para el Control de Enfermedades de los EE.UU (CDC) y el Centro Europeo para la Prevención y el Control de las Enfermedades (ECDC), recomiendan el uso de mascarillas N95 (Bartoszko et al.,).

Existe un informe de caso acerca de elementos de protección respiratoria para COVID-19, en donde 41 trabajadores de la salud fueron expuestos a procedimientos generadores de aerosoles producto de la atención a un paciente con neumonía grave, que posteriormente se confirmó como positivo para SARSCoV-2. Estos procedimientos realizados en la vía aérea incluyeron intubación endotraqueal, extubación, ventilación no invasiva y exposición a aerosoles en un circuito abierto. Todos los trabajadores de la salud expuestos al procedimiento dieron resultados negativos 14 días después de su fecha de exposición, a pesar de que $85 \%$ (35/41) usó máscaras quirúrgicas durante los procedimientos de alto riesgo ( $\mathrm{Ng}$ et al., 2020). De acuerdo con las pautas internacionales, debido a la situación que se está viviendo en el mundo y al stock de mascarillas requeridas, es fundamental considerar el uso de mascarillas N95 para procedimientos de alto riesgo cuando se generan aerosoles, sobretodo si existe escasez de estos (Bartoszko et al.,).

\section{Importancia de comprender el correcto sellado y manipulación de la mascarilla}

Se ha observado que la tasa de infección, cuando se emplean medidas estándar de protección personal, es considerablemente menor que cuando no se usan o se usan incorrectamente (Ran et al.; $\mathrm{Ng}$ et al.). Una de las razones más importantes para explicar el contagio en los trabajadores de la salud, está relacionada con la falta de mascarillas y/o el uso inco- rrecto de ellas (Wang et al., 2020; Ran et al.; Maxwell et al., 2020). Por lo tanto, es imprescindible la educación en el uso de los elementos de protección para que el equipo de salud utilice correctamente la mascarilla, cubriendo fosas nasales y boca, manipulando sólo de los elásticos y evitando tocar la zona anterior (Ministerio de Salud, 2020).

\section{Personal odontológico en contacto con pacientes COVID-19 positivos}

Para odontólogos y asistentes dentales que trabajan con pacientes sospechosos y/o infectados con COVID-19, debe considerarse el aislamiento obligatorio en las siguientes condiciones (Kowalski et al.,):

1. Aquellos con contacto cercano sin protección con pacientes COVID-19 positivo.

2. En caso de que presenten fiebre, tos, dificultad para respirar y otros síntomas (Debe aislarse de inmediato y recibir la atención adecuada).

Lo ideal es que al finalizar el trabajo en la sala de atención al paciente con COVID-19, se tomen muestras de hisopos nasofaríngeos u orofaríngeos para diagnosticar la enfermedad y posteriormente realizar un recuento sanguíneo completo. Aquellos profesionales que presenten resultados anormales en las pruebas deben someterse a un estricto aislamiento y observación, mientras que aquellos que se encuentren en condiciones normales, deben tomar un aislamiento para observación. Si no presenta ningún síntoma asociado a coronavirus, puede reanudar el trabajo dentro de una semana, aunque este periodo no ha sido definido con precisión (Kowalski et al.,; Chan et al., 2020a,b).

\section{CONCLUSIÓN}

La enfermedad por coronavirus ha aumentado progresivamente en la población chilena, donde un porcentaje considerable de los contagiados pueden ser asintomáticos. Lo anterior podría significar un alto riesgo para el personal odontológico, debido al trabajo crítico que se realiza en la cavidad oral en conjunto con la producción de gotas y aerosoles. Por lo tanto, es primordial tomar medidas básicas de protección para los pacientes y el equipo de salud, como lo son los elementos de bioseguridad (protectores oculares, faciales, mascarillas, gorros, bata y cubre-calzado), utilizar instrumentos esterilizables o desechables con un correcto protocolo de eliminación, desinfección de los equi- 
pos y superficies, en lo posible realizar procedimientos en una sala con entorno de presión negativa, considerando los tiempos de atención entre cada paciente y tomando las medidas de aislamiento necesarias posterior al contacto con pacientes COVID-19 positivo.

BOIN, B. C. \& MELIÁN, R. A. Dental care for COVID-19 positive patients. What to do in an emergency? Int. J. Odontostomat., 14(3):321-324, 2020.

ABSTRACT: Last December in China, an outbreak of pneumonia caused by a new viral strain of coronavirus or SARSCoV-2, never seen in humans, originated, which aroused worldwide attention. Likewise, on January 30 , a public health emergency of international concern was declared by the WHO, marking the start of a highly pathogenic pandemic called "2019 coronavirus disease (COVID-19)" that is transmitted from person to person by means of respiratory secretions. The main symptoms that appear in patients are fever, cough, sore throat, shortness of breath, fatigue and general malaise; However, there is a percentage of asymptomatic people who represent a high risk of contagion for health professionals who work in the airway and digestive tract, such as dentist. Consequently, this study aims to show the necessary protection measures so that the health team can intervene in patients diagnosed with COVID-19 safely, avoiding the spread of the disease.

KEY WORDS: COVID-19, dentistry, dental care, security.

\section{REFERENCIAS BIBLIOGRÁFICAS}

Bartoszko, J. J.; Farooqi, M. A. M.; Alhazzani, W. \& Loeb, M. Medical masks vs $\mathrm{N} 95$ respirators for preventing COVID-19 in health care workers a systematic review and meta-analysis of randomized trials. Influenza Other Respir. Viruses, 2020. DOI: https://www.doi.org/ 10.1111/irv.12745

Chan, K. W.; Wong, V. T. \& Tang, S. C. W. COVID-19: an update on the epidemiological, clinical, preventive and therapeutic evidence and guidelines of integrative Chinese-Western medicine for the management of 2019 novel coronavirus disease. Am. J. Chin. Med., 1-26, 2020a. https://www.doi.org/10.1142/S0192415X20500378

Chan, J. Y. K.; Wong, E. W. Y. \& Lam, W. Practical aspects of otolaryngologic clinical services during the 2019 novel coronavirus epidemic: an experience in Hong Kong. JAMA Otolaryngol. Head Neck Surg., 2020b. DOI: https://www.doi.org/10.1001/jamaoto.2020.0488

Coulthard, P. Dentistry and coronavirus (COVID-19) - moral decisionmaking. Br. Dent. J., 228(7):503-5, 2020. DOI: https://www.doi.org/ 10.1038/s41415-020-1482-1

Health and Safety Executive. Evaluating the protection afforded by surgical masks against infuenza bioaerosols: Gross protection of surgical masks compared to fltering facepiece respirators. 2008. Disponible en: https://www.hse.gov.uk/research/rrpdf/rr619.p.

Kowalski, L. P.; Sanabria, A.; Ridge, J. A.; Ng, W. T.; de Bree, R.; Rinaldo, A.; Takes, R. P.; Mäkitie, A. A.; Carvalho, A. L.; Bradford, C. R.; et al., COVID-19 pandemic: Effects and evidence-based recommendations for otolaryngology and head and neck surgery practice. Head Neck, 2020. DOI: https://www.doi.org/10.1002/hed.26164, 2020. doi:10.1002/ hed. 26164

Lai, T. H. T.; Tang, E. W. H.; Chau, S. K. Y.; Fung, K. S. C. \& Li, K. K. W. Stepping up infection control measures in ophthalmology during the novel coronavirus outbreak: an experience from Hong Kong. Graefes Arch. Clin. Exp. Ophthalmol., 258(5):1049-55, 2020.

Li, L. Q.; Huang, T.; Wang, Y. Q.; Wang, Z. P.; Liang, Y.; Huang, T. B.; Zhang, H. Y.; Sun, W. \& Wang, Y. COVID-19 patients' clinical characteristics, discharge rate, and fatality rate of meta-analysis. $J$. Med. Virol., 92(6):577-83, 2020.

Maxwell, D. N.; Perl, T. M. \& Cutrell, J. B. "The Art of War" in the era of Coronavirus Disease 2019 (COVID-19). Clin. Infect. Dis., 2020. DOI: https://www.doi.org/10.1093/cid/ciaa229

Meng, L.; Hua, F. \& Bian, Z. Coronavirus disease 2019 (COVID-19): emerging and future challenges for dental and oral medicine. J. Dent. Res., 2020. DOI: https://www.doi.org/10.1177/0022034520914246

Ministerio de Salud (MINSAL). Guias clínicas no ges- enfermedades trasmisibles, COVID-19. Santiago de Chile, Ministerio de Salud, Gobierno de Chile, 2020. Disponible en: https://diprece.minsal.cl/temasde-salud/temas-de-salud/guias-clinicas-no-ges/guias-clinicas-no-gesenfermedades-transmisibles/covid-19/recomendaciones/

Ng, K.; Poon, B. H.; Kiat Puar, T. H.; Shan Quah, J. L.; Loh, W. J.; Wong, Y. J.; Tan, T. Y. \& Raghuram, J. COVID-19 and the risk to health care workers: a case report. Ann. Intern. Med., 2020. DOI: https:// www.doi.org/10.7326/L20-0175

Ran, L.; Chen, X.; Wang, Y.; Wu, W.; Zhang, L. \& Tan, X. Risk factors of healthcare workers with Corona Virus Disease 2019: a retrospective cohort study in a designated hospital of Wuhan in China. Clin. Infect. Dis., ciaa287, 2020. DOI: https://www.doi.org/10.1093/cid/ciaa287

Remuzzi, A. \& Remuzzi, G. COVID-19 and Italy: what next? Lancet, 395(10231):1225-8, 2020.

Smith, J. D.; MacDougall, C. C.; Johnstone, J.; Copes, R. A.; Schwartz, B. \& Garber, G. E. Effectiveness of N95 respirators versus surgical masks in protecting health care workers from acute respiratory infection: a systematic review and meta-analysis. CMAJ, 188(8):567-74, 2016.

Ti, L. K.; Ang, L. S.; Foong, T. W. \& Ng, B. S. W. What we do when a COVID-19 patient needs an operation: operating room preparation and guidance. Can. J. Anaesth., 2020. DOI: https://www.doi.org/ 10.1007/s12630-020-01617-4

van Doremalen, N.; Bushmaker, T.; Morris, D. H.; Holbrook, M. G.; Gamble, A.; Williamson, B. N.; Tamin, A.; Harcourt, J. L.; Thornburg, N. J.; Gerber, S. I.; et al., Aerosol and surface stability of SARS-CoV-2 as compared with SARS-CoV-1. N. Engl. J. Med., 382(16):1564-7, 2020.

Wang, J.; Zhou, M. \& Liu, F. Reasons for healthcare workers becoming infected with novel coronavirus disease 2019 (COVID-19) in China. J. Hosp. Infect., 2020. DOI: https://www.doi.org/10.1016/ j.jhin.2020.03.002

Wu, Z. \& McGoogan, J. M. Characteristics of and Important Lessons From the Coronavirus Disease 2019 (COVID-19) Outbreak in China: Summary of a Report of 72 ?314 Cases From the Chinese Center for Disease Control and Prevention. JAMA, 2020. DOI: https:// www.doi.org/10.1001/jama.2020.2648

Zou, L.; Ruan, F.; Huang, M.; Liang, L.; Huang, H.; Hong, Z.; Yu, J.; Kang, M.; Song, Y.; Xia, J.; et al., SARS-CoV-2 viral load in upper respiratory specimens of infected patients. N. Engl. J. Med., 382(12):1177-9, 2020.

Zuo, M. Z.; Huang, Y. G.; Ma, W. H.; Xue, Z. G.; Zhang, J. Q.; Gong, Y. H.; Che, L. \& Chinese Society of Anesthesiology Task Force on Airway Management. Expert Recommendations for Tracheal Intubation in Critically ill Patients with Noval Coronavirus Disease 2019. Chin. Med. Sci. J., 2020. DOI: https://www.doi.org/10.24920/003724

Dirección para correspondencia:

Camila Boin Bakit

Facultad de Odontología

Universidad de Chile

Santiago - CHILE

Email: camila.boin@ug.uchile.cl

Recibido : 22-04-2020

Aceptado: 23-04-2020 